\title{
Niveles y distribución de metales pesados en el agua de la zona de playa de Riohacha, La Guajira, Colombia
}

\section{Levels and distribution of heavy metals in the water of the beach area of Riohacha, La Guajira, Colombia} Níveis e distribuição de metais pesados na água da área
da praia de Riohacha, Guajira, Colômbia

\author{
Carlos Doria Argumedo ${ }^{1}$ \& Haroldo Deluque Viloria ${ }^{2}$ \\ 'Licenciado en Química, Magister en Química, Estudiante de Doctorado en Ciencias Químicas, \\ Universidad de La Guajira. Riohacha. Colombia. ${ }^{2}$ Ingeniero Ambiental \\ 1,2 Facultad de Ingeniería. Programa de Ingeniería Ambiental. \\ Universidad de La Guajira Riohacha. Colombia. \\ 1'cdoria@uniguajira.edu.co, hdeluque@uniguajira.edu.co
}

\section{Resumen}

La zona de playa de Riohacha, La Guajira, Colombia, constituye un ecosistema muy rico, diverso y productivo, dotado de gran capacidad para proveer bienes y servicios que sostienen actividades como pesca, turismo y navegación; sin embargo, experimenta problemáticas ambientales producto de la dinámica de la ciudad, relacionadas con la infiltración de aguas residuales, acumulación de residuos sólidos orgánicos y descargue de sedimentos del rio Ranchería. En la metodología de esta investigación se tomaron muestras de agua de mar en cuatro puntos de un sector de aproximadamente $2.5 \mathrm{~km}$ de playa, durante ocho meses en el año 2013, en épocas de lluvia y sequía. Los metales analizados fueron Cobalto, Cobre, Zinc, Aluminio, Manganeso, Níquel y Hierro. Las concentraciones de los metales Ni y Zn, considerados muy tóxicos, se encontraron por encima de los niveles según la normativa nacional y algunas establecidas a nivel internacional, para efectos agudos en aguas marinas y estuarinas destinadas a la preservación de fauna y flora; las concentraciones de $\mathrm{Cu}$ y $\mathrm{Zn}$, se encuentran por debajo de los límites establecidos por la EEA y la EPA. La correlación significativa existente entre las concentraciones de los metales $\mathrm{Fe}, \mathrm{Mn}, \mathrm{Zn}$, Co con los nutrientes nitrato y fosfato, indica posible acumulación de ellos en el sedimento e incluso bioacumulación en la fauna asociada a esta zona. La presencia y concentraciones de los metales son producto, principalmente, de la intervención humana en las labores de minería y en el ciclo biogeoquímico de los metales.

Palabras clave: metales pesados, agua de mar, playa de Riohacha, concentración, nitrato, fosfato

\section{Abstract \\ The beach area of Riohacha, La Guajira, Colom- bia, is a very rich, diverse and productive ecosys- tem endowed with great ability to provide goods and services that support activities such as fis- hing, tourism and navigation; however, there are environmental problematics, product of the dyna- mics of the city, related to infiltration of wastewater,}


accumulation of organic solid waste and discharge of sediments of the Rancheria River. On the methodology of this research sea water samples were taken at four points of a sector of approximately $2.5 \mathrm{~km}$ from the beach, for eight months in the year 2013, in times of rain and drought. The metals analyzed were cobalt, copper, zinc, aluminum, manganese, nickel and iron. The concentrations of the metals $\mathrm{Ni}$ and $\mathrm{Zn}$, considered highly toxic, were found above the levels according to national rules and some established at international level, for acute effects in marine waters and estuarine intended for the preservation of fauna and flora; the concentrations of $\mathrm{Cu}$ and $\mathrm{Zn}$, are below the limits established by the EEA and the EPA. The significant correlation between the concentrations of $\mathrm{Fe}, \mathrm{Mn}, \mathrm{Zn}$, Co metals with nutrient nitrate and phosphate, it indicates possible accumulation of them in sediments and bioaccumulation in the fauna associated with this area. The presence and concentrations of metals are product, primarily, due to human intervention through mining and metals's biogeochemical cycle.

Key-words: heavy metals, sea water, beach of Riohacha, concentration, nitrate, phosphate

\section{Resumo}

A área da praia de Rioacha, Guajira, Colômbia, constitui um ecossistema rico, diverso e produtivo, dotado de grande capacidade para prover bens e serviços que sustentam atividades como pescaria, turismo e navegação; no entanto, experimenta problemáticas ambientais decorrentes da dinâmica da cidade relacionadas com a infiltração de águas residuais, acumulação de resíduos sólidos orgânicos e descarregamento de sedimentos do rio Ranchería. Na metodologia desta pesquisa, se tomaram amostras de água de mar em quatro pontos de uma área aproximada de $2.5 \mathrm{~km}$ de praia durante oito meses em 2013, tanto em épocas de chuva como de sequía. Os metais analisados foram cobalto, cobre Zinco, alumínio, manganês, níquel e ferro. Las concentrações dos metais $\mathrm{Ni}$ e Zn, considerados muito tóxicos, encontraram-se acima dos níveis permitidos segundo a normativa nacional e algumas estabelecidas a nível internacional, paras efeitos agudos em águas marinas e estuarinas destinas á preservação da fauna e flora. As concentrações de $\mathrm{Cu}$ e $\mathrm{Zn}$ encontramse abaixo dos limites estabelecidos pela EEA e a EPA. A correlação significativa existente entre as concentrações dos metais $\mathrm{Fe}, \mathrm{Mn}, \mathrm{Zn}, \mathrm{Co}$ e os nutrientes nitrato e fosfato, indica possível acumulação destes no sedimento e incluso bioacumulação na fauna associada a esta zona. A presença e concentrações dos metais são produto, principalmente, da intervenção humana em explotações mineiras e do ciclo biogeoquímico dos metais.

Palavras-chave: metais pesados, água de mar, praia de Rioacha, concentração, nitrato, fosfato

\section{Introducción}

Los factores causantes de mayor deterioro en los ecosistemas marinos costeros en Colombia se deben a fenómenos tanto naturales como antropogénicos, entre los que se encuentran una excesiva sedimentación, las anomalías climáticas, la acumulación de contaminantes y basuras, la interrupción de los flujos bioenergéticos, la sobreexplotación de los recursos, la utilización de artes de pesca altamente destructivas y las actividades turísticas (Garay et al., 2001). Específicamente las áreas costeras del departamento de La Guajira (norte de Colombia), presentan constante alteración de sus propiedades naturales como consecuencia del vertimiento continuo de desechos domésticos e industriales. Las aguas residuales domésticas carecen de tratamiento y son descargadas al mar a través de los ríos o directamente a éste, causando una importante disminución en la calidad de las aguas marino-costeras (UNEPRCU/CEP, 2010). En definitiva, una gran cantidad 
de compuestos químicos y mezclas complejas de éstos, son vertidos directamente al mar (PNUMA, 1999). La contaminación por metales pesados se ha constituido en una de las formas más peligrosas para los ecosistemas acuáticos, dado que son elementos poco o nada biodegradables, tienden a acumularse en los tejidos de animales y vegetales acuáticos, y permanecen en ellos por largos períodos, desencadenando procesos de biomagnificación y acciones toxicodinámicas, las cuales generan alteraciones metabólicas, mutaciones y transformaciones anatómicas en las especies animales, incluido el hombre (Panebianco, 2011).

Un renglón importante de la economía de la Guajira es la ganadería: vacunos, porcinos, equinos, mulares, asnales, caprinos y ovinos; la pesca, especialmente de mariscos, tortugas y perlas se realiza en forma artesanal. Además constituyen el hábitat de alimentación, anidación y descanso para diferentes especies marinas y un lugar de recolección artesanal de recursos marinos (Alcaldía Mayor de Riohacha, 2008). Es importante la explotación forestal de añil, caoba, cedro, dividivi, guayacán, mangle, roble, totumo. Carece de industria manufacturera (Espeleta, 2000). Según el censo 2005 (DANE, 2005) la actividad económica predominante es el comercio (52\%), el segundo renglón económico de la ciudad es el de los servicios (30\%), y luego hay otras actividades $(10 \%)$ y la Industria (8\%).

El departamento de La Guajira es una zona muy importante del Caribe colombiano, donde se realizan múltiples actividades socioeconómicas en diversos sectores productivos, que fomentan el desarrollo de su población. Estas actividades productivas y los asentamientos humanos generan residuos que, en mayor o menor medida, son las potenciales fuentes contaminantes del agua y los recursos naturales, si éstos no son manejados y dispuestos de forma adecuada para reducir el deterioro ambiental. La minería del carbón se presenta como una actividad que afecta de forma directa a los factores ambientales, con resultados críticos en los pastizales y praderas, la contaminación atmosférica, la diversidad de especies, las cadenas alimentarias, el uso y la contaminación del suelo y de los asentamientos antrópicos cercanos. Este tipo de minería se realiza a cielo abierto, produciendo impactos cuando se quita la cubierta de material estéril o capa vegetal que cubre la roca carbonífera para extraer el carbón, emitiendo partículas finas a la atmósfera; la mayor concentración está en la zona de explotación, los patios de acopio y las carreteras de ingreso a las mina; también se hacen descargas de agua de la minería hacia los cursos de agua natural; en épocas de lluvia, la escorrentía llega más rápido al río Ranchería sin regulación de caudales por no tener cobertura vegetal, sino una red de canales de drenaje, presentando efluentes con trazas de nitrato de amonio de la planta de explosivos, y aguas residuales de la planta de lavado de equipos con contenidos de grasas, aceites, trazas de combustible y carbón mineral.

Se presente investigación fue llevada a cabo para determinar la presencia de algunos metales pesados en las aguas de la zona de playa de la ciudad de Riohacha (La Guajira), con el objeto de conocer las rutas de estos contaminantes y su interacción con otras sustancias presentes en las aguas costeras. La zona investigada contiene ecosistemas muy ricos, diversos y productivos dotados de gran capacidad para proveer bienes y servicios que sostienen actividades como la pesca, el turismo, la navegación, el desarrollo portuario, la explotación minera y donde se dan asentamientos urbanos e industriales.

\section{Materiales y métodos}

El estudio fue realizado en la ciudad de Riohacha, ubicada en la parte central izquierda del departamento de La Guajira, latitud: 11'33’09.29" N - Iongitud: $72^{\circ} 54^{\prime} 33.20^{\prime \prime} \mathrm{O}$. Una altitud de $10 \mathrm{msnm}$, con $28^{\circ} \mathrm{C}$ y $35 \%$, de temperatura y humedad relativa promedio respectivamente. Esta área limita al norte con el mar Caribe, al oriente con el río Ranchería, los municipios de Manaure y Maicao, por 
el sur con los municipios de Hato Nuevo, Barrancas, Distracción, San Juan del Cesar y por el occidente con el municipio de Dibulla y el mar Caribe (Figura 1). La ciudad está situada entre la orilla del mar Caribe y la ribera occidental del delta del río Ranchería, específicamente del brazo El Riíto. El ecosistema corresponde a selva seca constituida por matorrales, bejucos, cactáceas y árboles de hojas compuestas, entre otros. Cerca de la costa, por efecto de desertización, el terreno es árido y el suelo arenoso. Al sur de la ciudad, el ecosistema es típico de sabana seca.
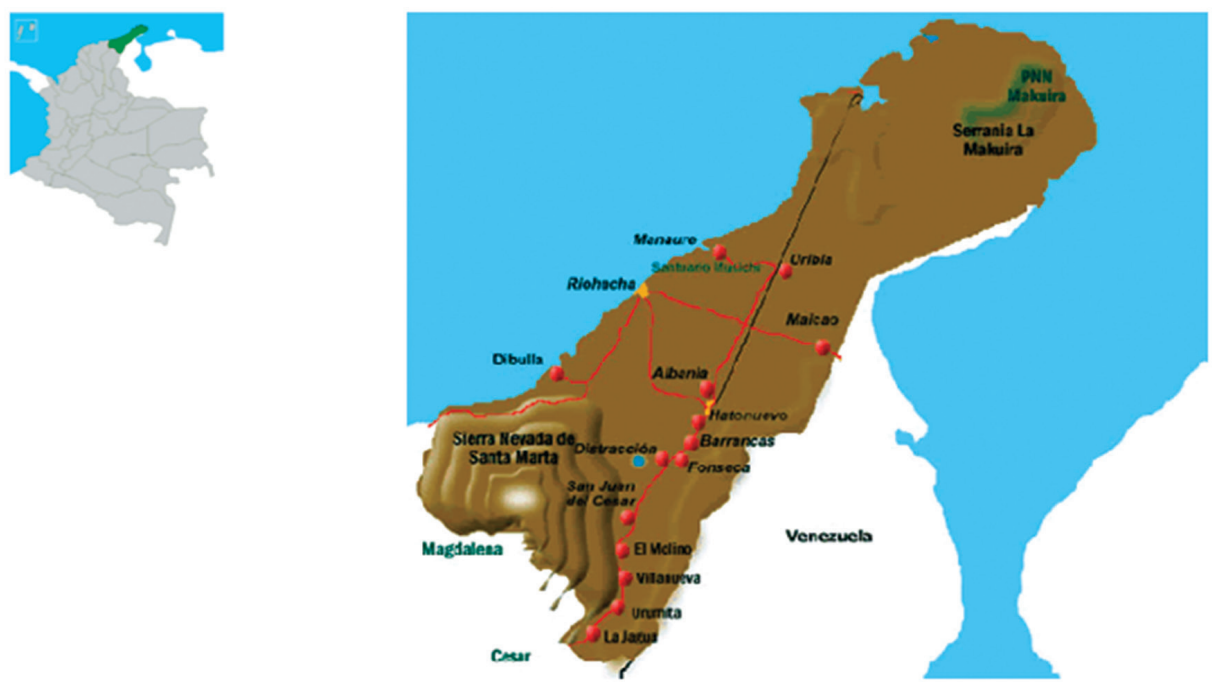

Figura 1. Ubicación geográfica de la ciudad de Riohacha (La Guajira, Colombia)

Entre marzo y diciembre del 2013 se realizaron un total de ocho campañas de seguimiento con dos puntos de muestreo en cada una de las cuatros estaciones investigadas a lo largo de un sector de aproximadamente $2,5 \mathrm{~km}$ de la playa de Riohacha (Figura 2).

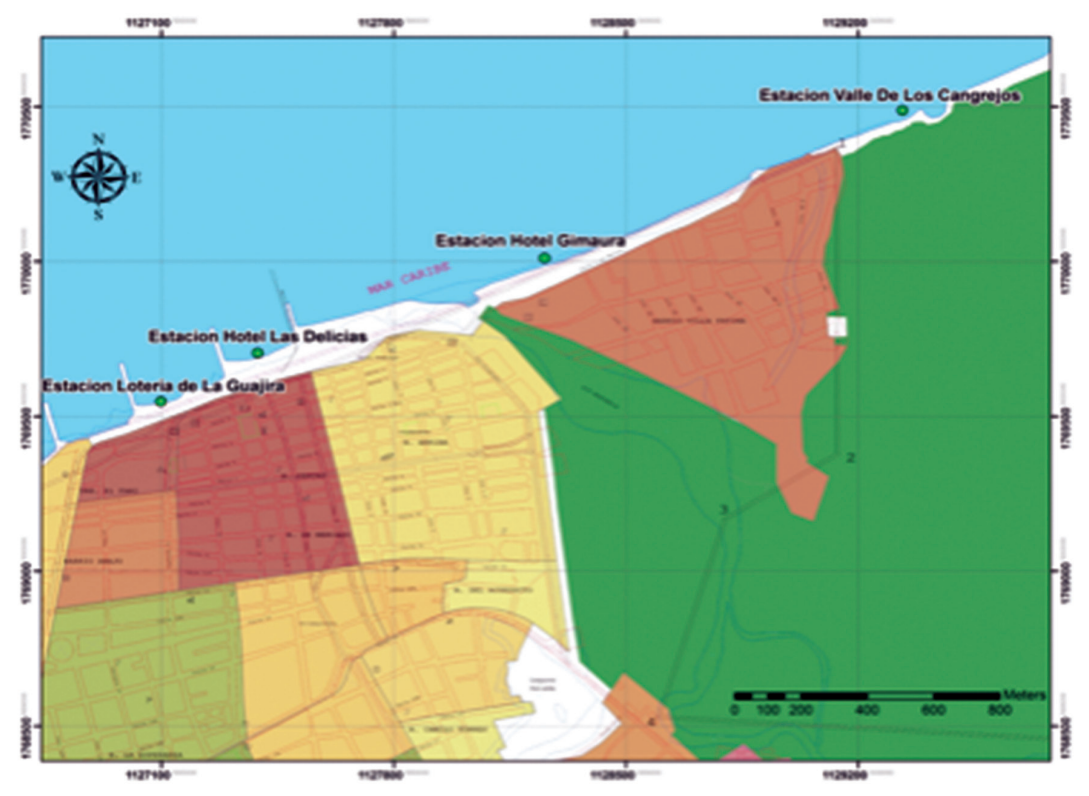

Figura 2. Estaciones de muestreo de metales pesados en la zona de playa de Riohacha (La Guajira, Colombia) 
Para el análisis de los metales se tomaron 500 $\mathrm{mL}$ de muestra mixta formada por tres submuestras colectadas a diferentes profundidades, una aproximadamente a $20 \mathrm{~cm}$ del fondo, otra a la mitad de la columna de agua y la última a $10 \mathrm{~cm}$ desde la superficie (Garay et al., 2003) Estas fueron colectadas con una botella muestreadora de fondo horizontal y almacenadas en frascos de polietileno previamente lavados con una solución al $5 \%$ de $\mathrm{HNO}_{3}$ y enjuagados con agua desionizada. En el laboratorio las muestras fueron filtradas al vacío (filtro Whatman de fibra de vidrio $0.45 \mu \mathrm{m}, 0.47 \mathrm{~mm} \varnothing$ y acidificadas con $\mathrm{HNO} 3$ hasta $\mathrm{pH} 2.0$ y refrigeradas a $4.0^{\circ} \mathrm{C}$ (APAHA-AWWA- WPCF, 2005), hasta posterior digestión y análisis por espectrofotometría de absorción UV-Visible (Espectrofotómetro $\mathrm{HACH}$ DR 5000). El muestreo para el análisis fisicoquímico fue realizado de la misma manera, pero sin acidificación. Se llevaron a cabo tres réplicas para cada muestra de agua. Los análisis realizados en campo correspondieron a los parámetros temperatura del agua, $\mathrm{pH}$, Oxígeno Disuelto, conductividad eléctrica y en el laboratorio se midieron los parámetros de Nitrato, Amonio, Fosfato y metales (Fe, $\mathrm{Cu}, \mathrm{Ni}, \mathrm{Mn}, \mathrm{Al}$, $\mathrm{Zn}, \mathrm{Co})$.

\section{Resultados y discusión}

Las condiciones fisicoquímicas del agua de mar en cuanto a los valores de $\mathrm{pH}$ con promedio de 8,22, oxígeno disuelto de $4,76 \mathrm{mg} / \mathrm{L}$ y conductividad de $57150 \mu \mathrm{S} / \mathrm{cm}$; son acordes con estos tipos de agua y considerados normales para esta región, además se encuentran dentro de los límites permisibles establecidos por la legislación colombiana (Decreto 1594 de 1984). Las concentraciones promedio de los metales en el agua de mar, obtenidas en la zona de muestreo de la playa de Riohacha se indican en la Tabla 1. La distribución en cuanto a la concentración de los metales pesados $(\mathrm{mg} / \mathrm{L})$ disueltos en las muestras en forma decreciente fueron: $\mathrm{Co}>\mathrm{Mn}>\mathrm{Fe}>\mathrm{Ni}>\mathrm{Zn}>\mathrm{Cu}$. Las concentraciones de $\mathrm{Cu}$ y $\mathrm{Zn}$ teniendo en cuenta la consideración como metales ligeros y tóxicos, se encuentran por debajo de los límites establecidos por la EEA y la EPA (EPA, 1999), los bajos niveles de estos metales en las aguas se explican por su baja solubilidad y la tendencia natural a precipitar y acumularse en los sedimentos (Perales, Garrido \& Ramírez, 2012). Las concentraciones de los metales $\mathrm{Ni}$ y $\mathrm{Zn}$ considerados muy tóxicos, aparecen por encima de los niveles para efectos agudos en aguas marinas y estuarinas para preservación de fauna y flora a nivel nacional (Ministerio de Salud, 1984) y referenciadas a nivel internacional (Buchman, 2008 y CONAMA, 1986).

Tabla 1. Concentraciones promedio ( $\mathrm{mg} / \mathrm{L})$ de metales en agua, en zonas de muestreo de la playa de Riohacha (La Guajira, Colombia)

\begin{tabular}{lcccc}
\hline \multicolumn{1}{c}{ Estaciones } & $\begin{array}{c}\mathrm{Al} \\
(\mathrm{mg} / \mathrm{L})\end{array}$ & $\begin{array}{c}\mathrm{Mn} \\
(\mathrm{mg} / \mathrm{L})\end{array}$ & $\begin{array}{c}\mathrm{Co} \\
(\mathrm{mg} / \mathrm{L})\end{array}$ \\
\hline E1 & & $<0.008$ & 0.112 & 0.500 \\
E2 & & $<0.008$ & 0.130 & 0.480 \\
\hline E3 & & $<0.008$ & 0.116 & 0.475 \\
E4 & & $<0.008$ & 0.113 & 0.479 \\
\hline Promedio & $\mathrm{Ni}$ & $\mathbf{Z n}$ & $\mathrm{Fe}$ & $\mathbf{C u}$ \\
\hline Estaciones & $(\mathrm{mg} / \mathrm{L})$ & $(\mathrm{mg} / \mathrm{L})$ & $(\mathrm{mg} / \mathrm{L})$ & $(\mathbf{m g} / \mathrm{L})$ \\
\hline E1 & 0.067 & 0.06 & 0.41 & 0.010 \\
\hline E2 & 0.074 & 0.04 & 0.08 & 0.016 \\
\hline E3 & 0.069 & 0.03 & 0.06 & 0.024 \\
\hline E4 & 0.056 & 0.04 & 0.04 & 0.036 \\
\hline Promedio & 0.066 & 0.04 & 0.14 & 0.021 \\
\hline
\end{tabular}

Las altas concentraciones de Mn pueden deberse a la desorción de este metal en la superficie del material particulado suspendido por el intercambio con los cationes $\mathrm{Na}^{+}$y $\mathrm{K}^{+}$presentes en el agua de mar, ya que el Mn se encuentra principalmente adsorbido en la superficie de las partículas o como oxihidróxidos reactivos (Martínez \& Senior, 2001). Márquez (1997) reporta distribuciones no conservativas de este elemento, señalando que las pérdidas de manganeso pueden ocurrir por oxidación 
del Mn(II) a Mn(IV) con la formación de material en suspensión durante la mezcla estuarina.

La presencia de los metales en el agua de mar tiene su origen antropogénico materiales incorporados por arrastre del río Ranchería (minería, usos de fertilizantes, aguas residuales domésticas), y de manera general por la intervención humana en el ciclo biogeoquímico de los metales. Riohacha y las poblaciones que conforman la cuenca del río Ranchería albergan un total de 244.804 habitantes los cuales producen 241 t/día de residuos sólidos, $43.260 \mathrm{~m}^{3} /$ día de aguas residuales domésticas cargadas con 2.955 t/año de materia orgánica en términos de $\mathrm{DBO}_{5}$ y de 5.909 t/año de $\mathrm{DQO}$, además con 709 t/año de nitrógeno; 47 t/año de fosfatos; 2.955 t/año de sólidos suspendidos totales y 1.2E+ 19 NMP/año de bacterias indicadoras de contaminación fecal. Las mayores cargas de contaminantes domésticas son producidas por la localidad de Riohacha que genera 1.928 t/año de $\mathrm{DBO}_{5}, 3.855$ t/año de DQO, 463 t/año de nitrógeno, 1.928 t/año de sólidos suspendido, 31 t/año de fósforo total y 7.7E+18 NMP/año de coliformes (Doria et al., 2013)

Según Otero (2013) el 40.5\% del suelo de la cuenca del río Ranchería está destinado para el uso ganadero, el $0.8 \%$ para uso agrícola, y el $1.5 \%$ para actividades mineras. En detalle, la minería se ha convertido en el principal eslabón de la economía guajira. Según los datos del PIB departamental de 2011, este rubro representó el 58\% del total del valor agregado producido en el departamento, dejando rezagados a otros rubros como la agricultura y diversas actividades industriales. (Otero, 2013)

Con base en la época de muestreo, se observa que las concentraciones de los metales en el agua de mar son mayores en el período seco (meses de junio y julio) que en el de lluvias, esto debido a que la reducción del volumen de agua facilita la solubilización de los metales y se concentran los iones y materiales en el sistema, al disminuir el caudal del río Ranchería. En detalle, el delta constituye la zona de amortiguamiento hidráulico del río Ranchería, considerado como uno de los cuerpos de agua de mayor importancia. Su caudal, directamente relacionado con la distribución anual de la precipitación, alcanza un valor mínimo anual de $2.9 \mathrm{~m}^{3} \mathrm{~s}^{-1}$ y un máximo de $30.0 \mathrm{~m}^{3} \mathrm{~s}^{-1}$, e incluso en los meses de marzo, abril, junio y agosto, la falta de lluvia hace que quede seco (Polania, Orozco \& Ángel, 2006).

El análisis de varianza realizado para las concentraciones de los metales en las épocas secas y de lluvias, muestra diferencias significativas para todos los metales $(p<0.05)$. Se observa una mayor concentración de los metales en la estación E1, a excepción del Cu que se concentra mayormente en la E4 (Figura 3), así mismo se observa que en la estación $\mathrm{E} 1$ el pH y las concentraciones de Nitrato y Fosfato son mayores que en el resto de la zona de la playa (Tabla 2, Figura 4), esta situación puede depender del hecho de que esta zona se caracteriza por el sistema manglárico y la influencia hídrica del brazo Calancala el cual forma pequeños estanques de agua en donde se observan procesos de degradación orgánica. Se tiene en cuenta además la disposición de residuos sólidos en la cuenca del río Ranchería; los vertederos de residuos sólidos constituyen otro elemento que impacta el ambiente porque perturban principalmente factores estéticos con importancia de afectación crítica para el uso y erosión del suelo, olor y visibilidad, la interfase aguatierra y comunidades cercanas. El $50 \%$ de los residuos sólidos generados en las viviendas son recogidos por el servicio de aseo, el $25 \%$ son quemados, el $14 \%$ disponen en patio, lote, zanja (llamados botaderos satélite), el $4 \%$ se entierra y el $7 \%$ es arrojado a cuerpos de agua. (Doria et al., 2013)

El pH básico de la E1 (8.60) afecta la especiación química y la movilidad de los iones, permitiendo así su mayor presencia en esta zona de la playa, ya que al aumentar progresivamente los iones $\mathrm{OH}$, los metales forman diferentes especies hidroxiladas solubles: $\mathrm{Zn}(\mathrm{OH})_{2} \mathrm{Co}(\mathrm{OH})_{2}, \mathrm{Co}(\mathrm{OH})_{3}, \mathrm{Fe}(\mathrm{OH})_{2}$, $\mathrm{Fe}(\mathrm{OH})_{3}, \mathrm{Mn}(\mathrm{OH})_{2}, \mathrm{Mn}(\mathrm{OH})_{4}$ (Marrugo \& Negrete, 2011) (Contreras, Mendoza \& Gómez, 2004). Las mayores concentraciones de Nitrato $(17.9 \mu \mathrm{g} / \mathrm{L})$ y Fosfato $(29.45 \mu \mathrm{g} / \mathrm{L})$ en la estación E1 indican la abundancia de materia orgánica en esta zona de 
la playa de origen vegetal, producto de la dinámica del manglar, y por ende mayor retención de los iones metálicos (ácidos húmicos, ácidos fúlvicos, quelantes) por la formación de complejos solubles e insolubles. Esta situación guarda concordancia con el estudio de Martinez, Senior \& Márquez (2006), el cual establece que las aguas del río Manzanares (Venezuela) ricas en materia orgánica de origen vegetal, al descomponerse producen ácidos húmicos tales como el fúlvico, el cual puede complejarse con los metales pesados que se encuentran libres e hidratados en el agua del ecosistema.

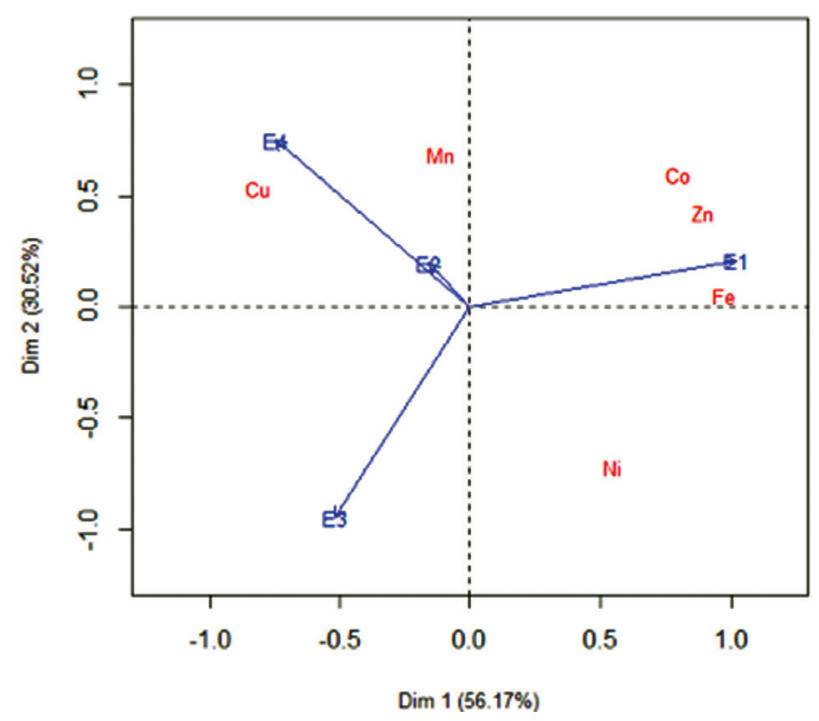

Figura 3. Distribución espacial de los metales en las zonas de muestreo de la playa de Riohacha (La Guajira, Colombia)

Tabla 2. Valores promedio de $\mathrm{pH}$ y concentraciones de nutrientes del agua de mar en zonas de muestreo de la playa de Riohacha (La Guajira, Colombia)

\begin{tabular}{llll}
\hline Estación & $\mathrm{pH}$ & $\begin{array}{r}\text { Nitrato } \\
(\mu \mathrm{g} / \mathrm{L})\end{array}$ & $\begin{array}{c}\text { Fosfato } \\
(\mu \mathrm{g} / \mathrm{L})\end{array}$ \\
\hline E1 & 8.60 & 17.90 & 29.45 \\
E2 & 8.47 & 14.30 & 17.10 \\
E3 & 8.40 & 10.58 & 25.00 \\
E4 & 8.40 & 6.08 & 15.34 \\
\hline Promedio & 8.46 & 12.21 & 21.72 \\
\hline
\end{tabular}

La concentración de Ni aparece con poca variabilidad en las diferentes estaciones de muestreo, debido a su gran capacidad de disolución en agua y la poca tendencia a formar complejos químicos con la materia orgánica, lo que permite su gran movilidad.

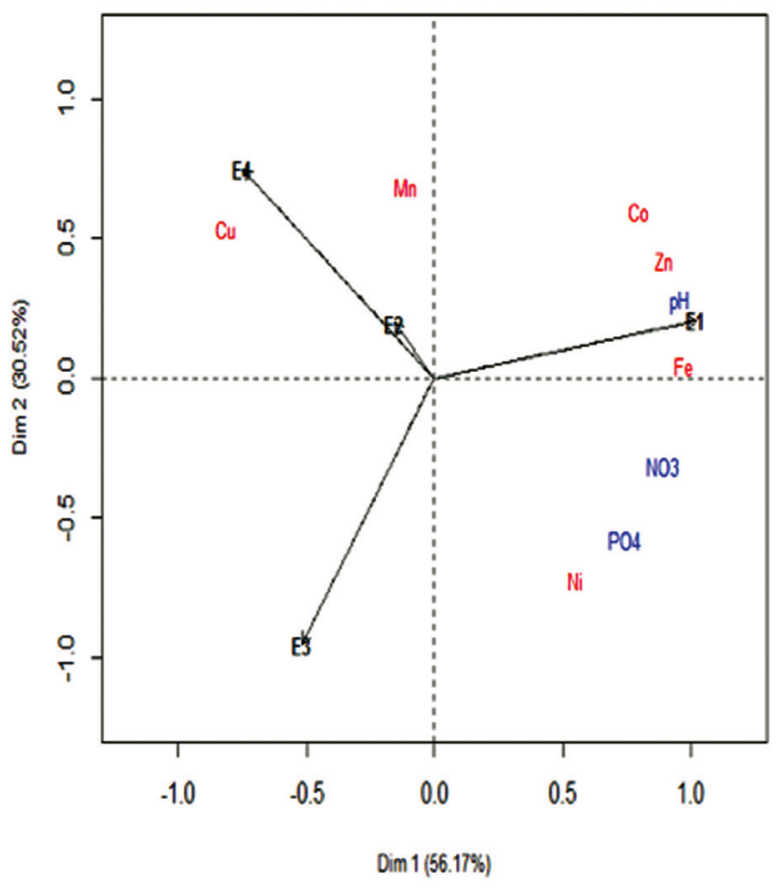

Figura 4. Distribución espacial de los metales, $\mathrm{pH}$ y nutrientes en las zonas de muestreo de la playa de Riohacha (La Guajira, Colombia)

El $\mathrm{pH}$ parece estar jugando un papel importante en la distribución y precipitación del zinc disuelto en la zona de la playa. Hahne \& Kroontje (1973) señalaron que este metal se hidroliza a pH mayores de 7.0, formando compuestos de hidróxido de gran estabilidad a pH superior a 8.0.

Los valores de $\mathrm{pH}$ del agua por encima de 7.0 influyen en la poca biodisponibilidad de Cu reflejándose en sus concentraciones bajas en comparación con los otros metales. Paulson, Curl \& Cokelet (1991) señalaron que la descomposición de la materia orgánica y el intercambio de iones controlan la liberación de alrededor de una tercera parte del Cu enlazado a partículas superiores a 53

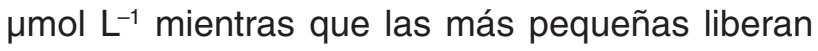
alrededor del $5 \%$ del Cu disuelto en agua de mar artificial, con concentraciones de metal muy bajas. 
Finalmente cabe destacar cómo la investigación realizada permite definir que existe un riesgo para la pesca y el hombre dado que los peces se alimentan, entre otras fuentes, de los nutrientes contenidos en los sedimentos. Así, los metales pesados contenidos en los sedimentos se bioacumulan en los peces ocasionando implicaciones serias para la salud de la franja de población que consume los productos de la pesca de esta zona. Además estos compuestos químicos sufren una amplificación biológica, es decir su concentración se incrementa a lo largo de la cadena trófica.

Se deduce también que las principales causas de contaminación son las actividades realizadas por la comunidad guajira en la cuenca del río Ranchería, donde se concentran los principales núcleos industriales y urbanos de esta región de Colombia. Así, es de gran interés e importancia para las autoridades ambientales, locales y regionales, establecer un programa de monitoreo y control continuo de la contaminación por metales en el medio marino y a lo largo del río Ranchería.

\section{Conclusiones}

La concentración de los metales $\mathrm{Ni}$ y $\mathrm{Zn}$ considerados muy tóxicos, se encuentran por encima de los niveles para efectos agudos en aguas marinas y estuarinas para preservación de fauna $y$ flora a nivel nacional (Decreto 1594 de 1984) y referenciadas a nivel internacional (Buchman, 2008; Conama, 1986).

La presencia de los metales en el agua de mar tiene su origen antropogénico por elementos incorporados por arrastre del río Ranchería (minería, usos de fertilizantes, aguas residuales domésticas)

La presencia y concentraciones de los metales es producto de la intervención humana en las labores de minería, en el ciclo biogeoquímico de los metales. Dentro de las actividades antrópicas, la minería de carbón a cielo abierto genera mayores impactos al medio ambiente que la minería subterránea; no solo afecta el interior de las minas, sino también a las áreas de influencia externas.

La correlación significativa existente entre las concentraciones de los metales Fe, Mn, Zn, Co con los nutrientes nitrato y fosfato, indica que puede haber acumulación de ellos en el sedimento e incluso bioacumulación en la fauna asociada a esta zona

La dinámica de metales pesados entre las estaciones estuvo sujeta a variaciones espaciales y temporales, como resultado de los cambios en la magnitud de la escorrentía terrestre, erosión del suelo y fuentes de contaminación metálica.

\section{Literatura citada}

1. Alcaldía Mayor de Riohacha. (2008). Plan de Desarrollo 2008-2011. Sector Agropecuario. Acuerdo N.005 de 2008. Oficina Asesora de Planeación Municipal. Riohacha

2. APAHA-AWWA- WPCF (American Public Healt Association Water Works Association and Water Polution Control Federation). (2005). Standard Methods for the examination of water and wastewater. 21Ed. New York.

3. Buchman, M.F. (2008). Screening Quick Reference Tables (SQuiRTs). NOAA OR\&R report 08-1 Seatle W.A, office of response and restoration division, national oceanic and atmospheric administration.

4. CONAMA-Consejo Nacional del Medio Ambiente de Brasil. (1986). Resolución CONAMA No 20 del 18 de junio de 1986.

5. Contreras, J., Mendoza, C. \& Gómez, A. (2004). Determinación de metales pesados en aguas y sedimentos del Río Haina. Ciencia y Sociedad. 2004, vol 29 (1), pp 38-71.

6. DANE- Departamento Administrativo Nacional de Estadística. (2005). Censo General 2005- información básica DNAE. Colombia. Recuperado de: http://www.dane.gov.co

7. Doria, C., Vivas, L., López, A., Deluque, H. \& Freile, E. (2013). Determinación de fuentes terrestres de contaminación en la zona costera de La Guajira. Colombia. Memorias VI Conferencia Internacional de Manejo Integrado de Zonas Costeras-CARICOSTAS. Santiago de Cuba. Cuba.

8. EPA. Enviromental Protection Agency of US. (1999). National Recommended Water Quality criteria Correction. U.S. EPA, 401M.Street, S.W. Washington.

9. Espeleta, B. (2000). La verdadera historia de Riohacha: el por qué Nicolás de Federmann no la fundó. Aarón Impresores. Riohacha

10. Garay, J., Marín, B., Ramírez, B., Vélez, A., Troncoso, W., Lozano, H., Acosta, J., Cadavid, B., Lancheros, A., 
Medina, O. \& Rondón, M. (2001). Diagnóstico y evaluación de la calidad ambiental marina en el Caribe y Pacífico Colombiano. Red de vigilancia para la conservación y protección de las aguas marinas y costera de Colombia REDCAM. Informe técnico final, INVEMAR. Santa Marta.

11. Garay, J., Ramirez, G., Betancourt, J., Marin, B., Cadavid, B., Panizzo, L., Lesmes, J., Sánchez, H. \& Franco, A. (2003). Manual de Técnicas Analíticas para la determinación de Parámetros y Físico-químicos y Contaminantes Marinos: Aguas, Sedimentos y Organismos. INVEMAR. Serie Documentos Generales No 13. Santa Marta

12. Hahne, H. \& Kroontje, W. (1973). Significance of $\mathrm{pH}$ and chloride concentration in behavior of heavy metals poIlutants: mercury (II), cadmium (II), zinc (II) and lead (II). J. Environ. Qual. 1973, vol 2, pp 444-448.

13. Márquez A. (1997). Comportamiento y distribución de algunos metales pesados en fracciones disueltas y particuladas en aguas superficiales del Río Manzanares, Estado Sucre, Venezuela. Tesis de pregrado, Licenciatura en Química, Escuela de Ciencias, Universidad de Oriente, Venezuela.

14. Marrugo J. \& Negrete, R. (2011). Evaluación de la contaminación por metales pesados en la Ciénaga La Soledad y Bahía de Cispatá, cuenca del Bajo Sinú, departamento de Córdoba. Facultad de Ciencias Básicas. Departamento de Química. Universidad de Córdoba. Montería.

15. Martínez, G., Senior, W. \& Márquez, A. (2006). Especiación de metales pesados en la fracción disuelta de las aguas superficiales de la cuenca baja y La pluma del río Manzanares. Estado Sucre. Venezuela. Ciencias Marinas. 2006, vol 32(2), pp 239-257.

16. Martínez, G. \& Senior, W. (2001). Especiación de metales pesados ( $\mathrm{Cd}, \mathrm{Zn}, \mathrm{Cu}$ y $\mathrm{Cr}$ ) en el material en suspensión de la pluma del Río Manzanares, Venezuela. Interciencia. 2001, vol 26, pp 53-61

17. Ministerio de Salud. (1984). Decreto No 1594 del 26 de Junio. Por el cual se reglamenta parcialmente el Título I de la Ley 9 de 1979, así como el Capítulo II DEL Título IV -parte III-Libro II y el Título III de la parte III - Libro I del decreto -Ley 2811 de 1974 en cuanto a usos del agua y residuos líquidos. Colombia.
18. Otero, A. (2013). Rio Ranchería: Entre la economía, la biodiversidad y la cultura. Documentos de trabajo sobre economía regional. Banco de la República. Centro de Estudios Económicos Regionales CEER. Cartagena.

19. Panebianco, M. (2011). Análisis de los niveles de metales pesados $(\mathrm{Pb}, \mathrm{Cu}, \mathrm{Cr}, \mathrm{Zn}, \mathrm{Ni}$ y $\mathrm{Cd})$ y aspectos reproductivos del delfín franciscana (Pontoporia blainvillei). Tesis doctoral. Universidad de Buenos Aires. Argentina. 2011.

20. Paulson Aj; Curl Hc Jr; \& Cokelet ED. (1991). Remobilization of $\mathrm{Cu}$ from marine particulate organic matter and from sewage. Mar.Chem. 1991, vol 33, pp 41-60.

21. Perales J., Garrido, C. \& Ramírez, M. (2012). Definición y desarrollo de controles de investigación para determinar la magnitud de la contaminación accidental y de los impactos producidos por episodios contaminantes en el litoral andaluz. Departamento de Ingeniería Química, Tecnología de Alimentos y Tecnologías del Medio Ambiente. Universidad de Cádiz. Informe final, Puerto Real.

22. PNUMA-Programa de Naciones Unidad para el Medio Ambiente. (1999). XII Reunión del Foro de Ministros de Medio Ambiente de América Latina y el Caribe. Bridgetown, Barbados.

23. Polanía J., Orozco, C. \& Ángel, I. (2006). Delta del Rio Ranchería (La Guajira, Colombia): Caudal, Salinidad y Transporte de sólidos y su posible influencia sobre composición y estructura de los manglares. Actual Biol. 2006, vol 28(84), pp 27-37.

24. UNEP-RCU/CEP. (2010). Actualización del informe técnico del PAC No 33 fuentes y actividades en la región del Gran Caribe. Cargas contaminantes domésticas e industriales y el aporte de las cuencas hidrográficas tributarias. Informe técnico del programa ambiental del Caribe UNEP-PAC-CIMAB.

\section{Conflicto de Intereses}

\author{
Los autores declaran no tener ningún conflicto \\ de intereses
}


